\title{
LA RAPPRESENTAZIONE DELLA VOLONTARIA NELLA GUERRA CIVILE SPAGNOLA ATTRAVERSO GLI ORGANI DI CONTROLLO FASCISTA
}

\author{
REPRESENTATION OF FEMALE VOLUNTEERS DURING SPANISH CIVIL WAR \\ THROUGH FACES SUPERVISORY BODIES
}

Arianna Fiore

Università di Firenze

\section{Resumen:}

La partecipazione femminile nella Guerra spanogla nel 1936 rappresenta la prima forma di consapevolezza politica e delle donne come genere. In Italia il fascismo ha monitorcreò una rete di informazioni e spionaggio capaci di controllare gli oppositori che participarono come volontari antifranchisti nella Guerra Civile spagnola. Ciò nonostante la descrizione della dissidenza femminile e della sua partecipazione nel conflitto molto più sulle categorie di genere che belle ragioni di determinazione e pericolosità politica.

\section{Palabras clave:}

donne volontarie, guerra civile spagnola, organi di controllo fascisti

\section{Abstract:}

Female participation in the 1936 Spanish Civil War represents the first form of political and awareness for women. Fascism in Italy created an information system to spy political opponents participating in the war against Franco. However, the representation of female, dissidents and of their participation in Spanish conflict was based much more on gender categories than on beliefs and political dangerousness.

\section{KeYwORDS:}

women, volunteers, Spanish Civil War, vigilance and repression of Anti-Fascism 
Da alcuni anni mi sto occupando, insieme a un gruppo di storici che studiano la partecipazione italiana antifascista alla guerra di Spagna, del ruolo avuto in questo conflitto dalle donne italiane ${ }^{1}$. Vorrei iniziare la presente riflessione con una premessa sulla questione delle fonti, di per sé abbastanza problematica. Il medievista Jacques Le Goff sosteneva che non esiste un documento innocuo e che ogni fonte deve essere interpretata non solo per quello che dice ma anche per quello che è, e quindi anche per chi l'ha prodotta, in quale contesto e con quale fine. Secondo la sua affermazione, è necessario porsi il problema delle fonti, posto che nessun documento deve essere considerato fonte di verità assoluta. Per studiare la storia dell'antifascismo si parte genericamente da due tipi di fonti: quelle provenienti dallo stesso mondo antifascista, che si descrive, si racconta, si interpreta, si ricorda o si celebra, o quelle prodotte invece dal Regime. In questo secondo caso, bisogna considerare fonti primarie i servizi d'informazione della polizia, le note della Regia Prefettura, le carte dei processidella Magistratura, le requisitorie dei Pubblici Ministeri presso il Tribunale Speciale per la Difesa dello Stato, i verbali degli interrogatori, ossia tutta la documentazione fornita o veicolata dai principali organi burocratici della struttura totalitaria. A questidocumenti di tipo ufficiale si aggiungono le fonti secondarie: delazioni prodotte da informatori, infiltrati, provocatori vari, indispensabile propaggine non istituzionale del Regime per la capillare sorveglianza dell'antifascismo. In entrambi i casi, come ricordavo poc'anzi, non si può confidare in un'assoluta oggettività del documento, sempre viziato dall'interpretazione di chi lo produce: gli antifascisti in un caso, gli organi istituzionali

-burocrati chiusi in un ufficio-e gli infiltrati, nel secondo.

Giampietro Nico Berti ritiene che per studiare l'anarchismo: “[...] le fonti di polizia sono indispensabili per ricostruire la cornice dei fatti. Quasi mai per interpretare il quadro esistente entro tale cornice" (Berti 2002: 17). Colgo la metafora, spostando il campo di analisi proposto da Berti per gli anarchici sulle donne: ritengo interessante vedere come il fascismo rappresentava la donna antifascista per poter approfondire non tanto il ruolo politico avuto da queste donne negli anni di opposizione (il quadro quindi), quanto ricostruirne la cornice, ossia la percezione che le istituzioni e i sistemi di controllo dell'apparato repressivo del Regime avevano del mondo femminile antifascista, continuamente sorvegliato, schedato, giudicato e condannato ${ }^{2}$.

1 Da queste ricerche è nato un saggio, Non avendo mai preso un fucile tra le mani, che nel corsodi un anno ha già goduto di una seconda pubblicazione con i nuovi risultati delle indagini, tra l'altro ancora in corso. Secondo indagini recenti, ad oggi ci si riferirebbe a un corpus che vede la partecipazione di novantatré donne italiane antifasciste nella guerra di Spagna, a cui bisognerebbe aggiungere quarantanove spagnole che assunsero la nazionalità italiana per essersi legate a volontari italiani. Il totale arriverebbe dunque a cento quarantatré.

22 Ricordiamo tra questi, oltre il Tribunale Speciale per la Difesa dello Stato, i fascicoli del Casellario Politico Centrale nell' Archivio Centrale dello Stato (d'ora in avanti ACS-CPC), la Divisione Polizia Politica, l'OVRA, l'Arma dei Carabinieri, la Milizia Volontaria per la Sicurezza Nazionale, il Partito Nazionale Fascista, il Bollettino delle Ricerche, l'iscrizione in Rubrica di Frontiera. 
Per fare questo mi sono affidata alle sole fonti fasciste, reperite presso il Casellario Politico Centrale dell'Archivio Centrale dello Stato (ACS-CPC), oggi fondamentali non soltanto per indagare il meccanismo repressivo dell'apparato giudiziario fascista ma anche, e soprattutto, il mondo dell'antifascismo ${ }^{3}$. Nel concreto si tratta di osservare come un determinato tipo di fonti ha descritto questo specifico nucleo di oppositori al fascismo, caratterizzato per genere, trattandosi di sole donne, per nazionalità, restringendo il corpus alle sole italiane, e per l'evento che le coinvolse, il conflitto spagnolo del 1936- 1939. I limiti temporali della ricerca s'identificano quindi con quelli del ventennio del Regime fascista (1922-1943), quando queste donne esplicano la loro attività militante.

Mussolini aveva previsto una corretta maniera di essere italiano, che coinvolgeva non solo l'ambito pubblico, quindi generalmente politico, ma anche quello privato, ampliando quindi notevolmente il suo raggio d'influenza. Bisognava fascistizzare la nazione e ciò doveva avverarsi contemporaneamente nella rete capillare della società, composta alla base dalla famiglia e dai singoli individui che la formano, uomini e donne. Nel caso femminile, ossia, per "fare le italiane", il rapporto tra pubblico e privato assume una proporzione diversa rispetto a quanto avveniva con la popolazione maschile. Il risultato fu avvicinare sempre più i due ambiti, dando vita a quello che Giovanni De Luna definisce "una commistione pubblico/privato destinata a durare nel tempo, ben al di là della caduta del fascismo" (De Luna 1995: 36). Le donne, inserite nelle varie corporazioni femminili, come l'Opera Nazionale Balilla, i Fasci femminili e l'Opera Nazionale per la Protezione della maternità e dell'infanzia (OMNI), destinata alla "difesa e il miglioramento fisico e morale della razza" (Mazza 1934: 11), erano sottoposte alla tutela dello Stato dalla nascita alla morte. Il fascismo contribuì sicuramente ad ampliare la presenza della donna nella sfera pubblica,senza però che ciò implicasse dei cambiamenti nella condotta che lei doveva tenere nella sfera privata, e mantenendo quindi inalterati i ruoli assegnati dalla tradizione madre, moglie, sorella e figlia- e le caratteristiche che doveva contemplare -fecondità, modestia, docilità, obbedienza, decoro, contegno, quando non rassegnazione e spirito di sacrificio. Al potere dell'uomo, soggetto attivo, corrisponde la sottomissione della donna, oggetto passivo, e l'idealizzazione della madre, giacché il fascismo fece della maternità un dovere nazionale.

33 Le donne schedate nel CPC sono 5005: 2.260 sono considerate antifasciste, 1.605 comuniste (più altre due su cui c'è dell'incertezza), 499 socialiste, 387 anarchiche (anche in questo caso c'è un'altra donna sospettata di essere anarchica), cinquanta repubblicane, venticinque antinazionali, cinque sovversive, una autonomista, una massona e una definita genericamente sospetta. È necessario ricordare che non tutte le donne oppositrici del fascismo vennero schedate nel CPC. Numerose donne che presero parte alla guerra civile spagnola non hanno una scheda personale. Molte donne rientrarono tra i nomi schedati dal Bollettino delle Ricerche e iscritte in Rubrica di Frontiera solo per essere andate clandestinamente all'estero, in Francia, Belgio e, nel nostro caso, in Spagna, a combattere o a sostenere la causa repubblicana. 
L'applicazione del principio del 'ciascuno al suo posto' assumeva come priorità strategica la conferma della netta opposizione tra una cittadinanza piena riconosciuta agli uomini in quanto titolari di una partecipazione attiva alla sfera pubblica e la passività della sfera domestica e privata che competeva alle donne (De Luna 1995: 45).

Cosa era dunque vietato alla donna in epoca fascista? Principalmente la politica. Già nel 1921, Mussolini aveva affermato a proposito della questione elettorale:

Non darò il voto alle donne. La donna deve ubbidire. La mia opinione della sua parte nello Stato è opposta a ogni femminismo. Naturalmente non deve essere schiava, ma se le concedessi il diritto elettorale, mi si deriderebbe. Nel nostro Stato essa non deve contare ${ }^{4}$.

A prescindere da abili strategie comunicative per accattivarsi il consenso delle italiane meno condiscendenti, in sostanza la sua opinione cambiò poco se ancora nel 1939, il Decalogo della piccola italiana ricordava alle ragazze che "La patria si serve anche spazzando la casa". Altro ambito di competenza esclusivamente maschile era la guerra. Secondo Marinetti, "La guerra dobbiamo amarla con tutto il nostro cuore di maschi", assegnando alla donna solo una funzione biologica: "La guerra sta all'uomo come la maternità sta alla donna". Un decreto del 1927 inoltre aveva dimezzato gli stipendi femminili, scoraggiando quindi la partecipazione femminile anche al mondo del lavoro: macchine e donne erano considerate dal Duce gravi cause di disoccupazione. L'ideologo del fascismo, Ferdinando Loffredo, nel 1938 non lasciò adito al dubbio:

L'abolizione del lavoro femminile deve essere la risultante di due fattori convergenti: il divieto sancito dalla legge, la riprovazione sancita dalla opinione pubblica. La donna che -senza la più assoluta e comprovata necessità- lascia le pareti domestiche per recarsi al lavoro, la donna che, in promiscuità con l'uomo, gira per le strade, sui tram, sugli autobus, vive nelle officine e negli uffici, deve diventare oggetto di riprovazione, prima e più che di sanzione legale (Loffredo 1938: 365$)^{5}$.

Tale concetto, tra l'altro, era stato già difeso da Pio XI. In effetti, nel 1931, nell'enciclica

4 Si veda a questo proposito: http://www.ciusa.it/attachments/article/455/Documentazione\%20 del\%20Coordinamento\%20Donne\%20dell'A.N.P.I.\%20di\%20Nuoro.pdf

55 Ferdinando Loffredo riprese la suddivisione lombrosiana delle donne in delinquente, prostituta e donna normale, basandosi sull'inferiorità del genere dovuta a un cervello meno pesante, che comporterebbe un'inferiorità, una minore intelligenza e sensibilità, una maggiore propensionealla menzogna e alla crudeltà della donna rispetto all'uomo. Inoltre, avvicinandosi ai precetti della Chiesa, secondo l'ideologo fascista una sessualità non finalizzata alla procreazione sarebbe del tutto deprecabile in quanto corrisponderebbe all'istinto criminale di prostitute, femministe e delinquenti. Il 31.12.1930, con l'Enciclica Casti connubii Pio XI aveva sostenuto la santità del matrimonio e la sua finalità procreativa. 
Quadragesimo anno, il Papa scriveva: Le madri di famiglia prestino l'opera loro in casa soprattutto o nelle vicinanze della casa, attendendo alle faccende domestiche. Che poi le madri di famiglia, per la scarsezza del salario del padre, siano costrette ad esercitare un'arte lucrativa fuori delle pareti domestiche, trascurando così le incombenze e i doveri loro propri, e particolarmente la cura e l'educazione dei loro bambini, è un pessimo disordine, che si deve con ogni sforzo eliminare ${ }^{6}$.

Per non parlare della cultura e dell'istruzione ${ }^{7}$, che avrebbero deviato la donna dalle sue occupazioni e dai piaceri della famiglia. Stanis Ruinas, nel 1930, esaltando ladonna fattrice d'italiani, la macchina da riproduzione, affermò che la donna che scrive,-la donna colta, da lui definite con scherno "scribacchina" - non sarebbe altro che "una donnetta che per soddisfare la propria vanità passa sopra ogni legge morale", molto "al disotto della prostituta", e ribadisce di non aver alcun rispetto "per la mediocre donna di lettere, che sciupa il tempo e sacrifica se stessa non per concorrere al potenziamento della Nazione ma per favorire la disgregazione della sua compagine morale" (Ruinas 1930: 36).

Pertanto, per la donna antifascista, la propria scelta ideologica implicava non soltanto una partecipazione politica attiva, seppur clandestina, ma anche una ridefinizione del proprio ruolo di donna, in ambito pubblico e, soprattutto, privato. Essere antifasciste voleva dire non solo condurre la lotta politica contro il fascismo, ma anche vivere in maniera antagonista rispetto a quanto era prospettato dal Regimea proposito di vita di coppia, sessualità, amore, figli, rapporto con i genitori, con la famiglia, tempo libero, cultura, letture, il proprio corpo (includendo moda, trucco, capelli). Se gli uomini antifascisti erano condannati per la loro opposizione politica, quindi per la loro particolare partecipazione da antagonisti nella sfera pubblica imposta dal regime, l'antifascismo femminile venne accusato spesso non tanto per la sua pericolosità ideologica, quanto piuttosto per i modi in cui le donne vivevano la sfera privata, dando quindi particolare importanza a "l'inquietudine, la ribellione, la dissimulazione, lo scetticismo e una consapevolezza crescente dei loro diritti di donne e di cittadine" (De Grazia 1993: 31). Come sostiene Alessandra Gissi, "una donna solita a condurre un'esistenza non del tutto aderente a modelli imposti dal fascismo poteva essere ritenuta una sovvertitrice degli ordinamenti dello Stato" (Gissi 2002: 37) ${ }^{8}$.

6 http://www.vatican.va/holy_father/pius_xi/encyclicals/documents/hf_p-xi_enc_19310515_ quadragesimo-anno_it.html (consultato il 3.12.2014).

7 Nel 1924, sul "Popolo d'Italia" Giuseppe Pochettino si complimenta con il ministro Gentile per le sue iniziative sull'educazione della donna: "lodo senza restrizioni il ministro Gentile, che, istituendo il Liceo femminile, ha creato quella che definirei la scuola di un sano femminismo, perché la scuola che veramente prepara la donna quale la natura e la ragione la vogliono, la donna cioè che, entrando domani nella società, vi prenderà il suo posto d'onore senza urti, senza antagonismi, senza rancori perché prenderà il posto di regina della casa, quello che veramente le compete" (Meldini 1975: 75).

8 Alessandra Gissi, Un percorso a ritroso: le donne al confino politico (1926-1943), in "Italia contemporanea", n. 226, marzo 2002. 
I ritratti delle donne avverse al regime offerti dal Casellario Politico Centrale si concentrano infatti sulla loro distanza rispetto al modello femminile proposto dal Regime, la massaia rurale, la giovane italiana, la custode del focolare e la fattrice di italiani (Guerrini 2013: 12-14). Le schede biografiche mettono in luce diversi elementi: gli aspetti fisiognomici, la condotta morale e civile, il livello culturale, le dicerie, la situazione sentimentale e familiare, le letture, l'attività sovversiva. La descrizione è basata su elementi fortemente discriminanti a livello di genere. Secondo Martina Guerrini ciò dipende da chi scriveva detti documenti: burocrati fascisti di genere maschile:

Gli uomini preposti alla classificazione e all'allestimento delle biografie sovversive hanno un posizionamento ben definito: eterosessisti con forte pregiudizio omofobo, membri della classe politica dominante, fiduciosi nel ruolo disciplinante della famiglia autoritaria -non a caso oggetto di continui rimandi e definizioni da parte di Mussolini- così come nel ruolo dello Stato, del paternalismo patriarcale e violento fascista, della funzione pedagogica del Partito (Guerrini 2013: 28).

Per riprendere la metafora di Nico Berti, vorrei aggiungere due notizie relative al quadro. Premetto che non tutte le italiane che presero parte alla guerra di Spagna, poco meno di un centinaio escludendo le spagnole che acquisirono la nazionalità dopo le nozze con volontari italiani, sono schedate al CPC, ma solo trentacinque di loro'. In Spagna ebbero un ruolo attivo, all'inizio combattendo in prima linea, dopo lo scioglimento delle milizie nelle retrovie, prestando servizio infermieristico e medico, occupandosi dei bambini, dell'istruzione, dell'approvvigionamento, della cucina. Appartengono a ogni classe sociale, con i livelli culturali più diversi. Spesso andarono in Spagna insieme al proprio compagno, anche se in alcuni casi la loro partecipazione ha cause diverse, come nel caso del collettivo di sette donne mandate dal PCI.

Ma arriviamo alla cornice, che è quello che in questa sede mi interessa. La narrazione offerta dalle fonti fasciste si gioca su due fronti per un certo senso contraddittori ${ }^{10}$. Nel

9 Ringrazio Andrea Andrico, Gianpaolo Giordana e Augusto Cantaluppi per avermi aiutato a reperire il materiale utilizzato nel presente saggio e per i loro indispensabili consigli.

10 Nel tentativo di fascistizzare la società, il Regime si avvalse anche di un lessico specifico che ne riflettesse l'ideologia. Maschile e femminile vennero contrapposti, connotando il primo i valori positivi, il secondo quelli negativi. La mascolinità era intesa come forza vitale, e quindi tutto quello che doveva essere elogiato diventava "maschio": troviamo una "voce maschia", un "temperamento maschio", "la forza maschia", "una maschia espressione", "il maschio vigore", un "maschio cameratismo", "la maschia speranza" e"la maschia prole". Anche la natura assume connotati maschili: "Com'è virile il cielo, oggi!", pensava il protagonista de Forse che sì, forse che no di D'Annunzio. Mussolini è la summa della virilità: è "l'Uomo più grande che l'Italia ha", "Mai nessuno lo vide piangere", "Egli non conosce stanchezza", "un Uomo così non s'era mai visto". Dal lato opposto, abbiamo l'antitesi della forza e della virilità: gli avversari, gli antifascisti, che sono definiti "fiacchi", "intorpiditi", "occhialuti", "pupattoli", "infrolliti", "meschini", "biechi", "depravati", "degenerati" ma, soprattutto, "femminette". A proposito del lessico del fascismo si veda il saggio di Giovanni Lazzari Le parole del Fascismo, la prima ricerca organica sul linguaggio del ventennio, in cui la lingua è intesa come specchio della realtà, in corrispondenza con l'ideologia. 
primo il narratore non esce dallo schema tipico che vuole la donna succube, essere inferiore all'uomo. La miliziana è interpretata come l'amante del repubblicano, pur essendone eventualmente la moglie; non ha idee proprie, ma va in Spagna imbevuta degli ideali del proprio uomo o della propria famiglia. La sua militanza non è basata su convinzioni politiche: è un qualcosa che viene recepito e assimilato, docilmente. L'autodeterminazione e la coscienza politica non sono nemmeno prese in considerazione come possibilità. La donna ritratta, in un certo qual modo, rientra nei canoni imposti dal Fascismo: è remissiva, sottomessa al suo uomo, non pensante.

Maria Piras "serbò regolare condotta morale e politica fino al 1921, epoca in cui contrasse matrimonio col noto comunista Polano Luigi. Appena diventata la moglie di costui, la Piras cominciò a seguirne le direttive politiche, apprendendo da lui le dottrine comuniste" (ACS-CPC, b. 3997) ${ }^{11}$, Ada Grossi "frequenta all'estero la compagnia dei sovversivi, seguendo l'esempio del padre socialista schedato" (ACS-CPC, b. 2540), e anche sua madre Maria Olandese "all'estero frequentava la compagnia dei sovversivi seguendo l'esempio del marito e dei figli" (ACS-CPC, b. 3583). Di Teresa Noce si dice che "Nel 1923 conobbe il noto comunista Longo Luigi di Giuseppe, da Fubine, del quale divenne l'amante e condivise le idee politiche", che "Seguì il marito in Spagna" e che si troverebbe là per "coadiuvare il marito nelle funzioni di Commissario Politico della 12 Brigata Internazionale" (ACS-CPC, b. 3553). Camilla Restellini, già "antifascista e seguace delle idee sovversive della madre", " professa idee socialiste, ed è scaltrissima, ha accompagnato sempre il Bassanesi nelle sue peregrinazioni all'estero e ha preso attiva parte alla propaganda sovversiva che lo stesso esplicava sia in Francia, sia nel Belgio che nella Spagna" (ACS-CPC, b. 4288). Rita Montagnana, riconosciuta leader femminile del PCI, venne iscritta in Rubrica di Frontiera solo come "moglie del comunista Togliatti, da fermare" (ACS-CPC, b. 3358), e stupisce il burocrate fascista per il suo abbigliamento elegante, proprio negli anni in cui Carlalberto Grillenzoni, nell'articolo "È brutta e sciamannata: sarà di sicuro prolifica", rilevava statisticamente che "L'eleganza nel vestire e, in ogni modo, la cura della propria toilette influiscono molto sfavorevolmente su la fecondità" (Meldini 1992: 192).

Parlavo di contraddizione. Se da una parte abbiamo la donna mite e docile, dall'altra abbiamo una narrazione che ritrae una donna spietata, che sovverte il mito della donna madre, moglie, angelo del focolare, prima confermato. Paolina Rocchetti è definita "fanatica sovversiva piena di odio verso il Regime e il Duce" (ACS-CPC, b. 4364), e l'anarchica Tosca Tantini, in una lettera intercettata spedita alla sorella del defunto amante Gualandi Bruno, promette addirittura di vendicare la sua morte (ACS-CPC, b. 5024).

11 La documentazione a cui mi riferisco è stata reperita presso il Casellario Politico Centrale dell'Archivio Centrale dello Stato di Roma (ACS-CPC). Nell'indicazione bibliografica viene incluso anche il riferimento alla specifica busta (b.) relativa alla donna sorvegliata. 
Per completare questo ritratto antagonista di donna, si aggiungono non di rado altre caratteristiche non contemplate nel modello femminile imposto dal Regime: nella sfera privata vediamo che alla fedeltà si oppone una certa dose di libertinismo, troppa facilità nel cambiare partner, o piuttosto la propensione a non essere madre, o a non esserlo in modo esemplare. Se non sono sposate, le antifasciste spesso vengono definite "dedite alla prostituzione", pur non avendolo mai fatto. La definizione è valida anche per le donne sposate che hanno seguito un altro uomo: la loro unione è sempre rimarcata da verbi che ne sottolineano l'illegittimità. Di Rosa Cremoni si legge: "Sposatasi all'età di 16 anni con un suddito belga, non è rimasta col marito neppure un anno. Sarebbe donna di facili costumi e, secondo quanto mi è stato riferito, avrebbe viaggiato in Germania, Polonia e Svizzera" (ACS-CPC, b. 1527). Maria Lombardi "non risulta che abbia contratto matrimonio col sovversivo Lorenzoni Aldo con il quale convive" (ACS-CPC, b. 2819). Fosca Corsinovi è indicata come "già amante del noto anarchico Barbieri", e poi "secondo notizie fiduciarie [...] convivrebbe con il nominato Nemo o Memmo, già residente negli S.U. d'America nonché già amante della pure nota Simonetti Maria di Giovanni" (ACS-CPC, b. 1489). Giovanna Zanarini "risulta trovarsi attualmente a Parigi, dove si è unita e fa vita comune col noto pericoloso comunista fuoriuscito Zanelli Ezio fu Amleto" (ACS-CPC, b. 5510), Giuditta Zanella “È associata da parecchi anni al noto anarchico schedato Margarita Ilario, col quale lotta costantemente per l'ideale anarchico" (ACS-CPC, b. 5516), mentre Siberia Gilioli "figlia dell'anarchico Onofrio, e amante dell'anarchico Cavani Renzo, si troverebbe ora a Port Bou insieme al noto Bruna Efisio, di cui sarebbe l'amante" (ACS-CPC, b. 2412). Lucia Minon "Prima di maritarsi veniva indicata quale donna di facili costumi avendo contratte varie relazioni intime" (ACS-CPC, b. 3304). Leonetta Mazzini “Viveva maritalmente con un suddito portoghese" (ACS-CPC, b. 3181), Egle Sani viene definita "di cattiva moralità, tanto che fu anche scacciata dal tetto coniugale [...] dedita ai piaceri e alla crapula" (ACS-CPC, b. 3181), mentre Elettra Pollastrini "ha ammesso di aver vissuto maritalmente per sette anni con il noto fuoriuscito Marchetti Virgilio", ma è comunque "Ritenuta ragazza poco seria, amante dei balli e dei divertimenti" (ACS-CPC, b. 4066). Per non parlare di Anna Launaro, che dopo aver convertito al comunismo il marito, nel 1923 abbandonò tetto coniugale, coniuge e figlio “[...] per recarsi all'estero con l'amante Quaglierini Giuseppe (sic) noto anarchico (sic)" (ACS-CPC, b. 2732), (in realtà si chiamava Vittorio ed era comunista). Quindi, vediamo una donna che non solo non è docile, ma che spesso seduce l'uomo, ha una funzione dominante nella coppia e lo spinge a compiere azioni sovversive: Eugenia Lina Simonetti, che si dice essere "di dubbia condotta morale e di sentimenti comunisti", pare "probabile che [...] abbia rafforzato nello Schirru l'idea dell'attentato" (ACS-CPC, b. 4822), mentre Luisa Zapatero: "Viene descritta come donna di carattere che domina il marito il noto Tibiletti Luigi” (ACS-CPC, b. 5544). 
È quindi sotto questa veste, come donne "abili simulatrici", "squilibrate", "suggestionabili" e "nevrotiche", che partecipano alla politica e prendono parte alla guerra" ${ }^{12}$. Maria Bibbi viene descritta per il suo "carattere piuttosto impulsivoed arrogante" e per essere una "donna scaltra, intelligente ed abile" che "non si farebbe sfuggire alcuna occasione per eventuali azioni ostili al Regime del qualeessa è un'irriducibile nemica" (ACS-CPC, b. 635), Emma Bronzo viene definita per aver tenuto un "contegno tipicamente e rigidamente comunista" (ACS-CPC, b. 851). Un telegramma registra che Virginia Gervasini, "pericolosa sovversiva, amante pericolosissimo comunista sinistra Di Bartolomeo Nicola, est partita da Spagna vuolsi diretta Francia et poscia Regno onde svolgere incarichi natura politica" (ACS-CPC, b. 2347), Maria Giaconi: "svolse sempre attività sovversiva, tanto da essere considerata la direttrice di tutte le attività antitaliane che avevano luogo in detta città" (ACS- CPC, b. 2378). Emilia Belviso "risulta essere stata impiegata presso la Delegazione Urss di Genova e sarebbe stata intermediaria per la Liguria del Soccorso Rosso verso i condannati politici" (ACSCPC, b. 478), Giuditta Zanella "Ha sempre preso partea manifestazioni sovversive, e fu più volte arrestata pel suo carattere ribelle" (ACS- CPC, b. 1489) mentre Leonetta Mazzini viene arrestata con l'accusa "di aver incitato nell'aprile 1937 le milizie rosse ad assaltare le carceri di Calle Duque Sexto ed uccidere tutti i prigionieri che vi si trovavano [...] di aver servito di collegamento nelle predette carceri per denunziare le famiglie dei prigionieri stessi" (ACS-CPC, b. 3181).

La donna viene stigmatizzata dunque perché dimostra di avere idee politiche, un ruolo all'interno del mondo antifascista, al pari dei maschi, e perché è colta, sa parlare durante i meeting, scrive su fogli politici, legge. Se di Fosca Corsinovi si dice infatti essere "Di sufficiente cultura e viva intelligenza", Giuditta Zanella "intelligente ma di poca cultura", tuttavia "Fa propaganda fra la classe operaia femminile, con profitto. Ha preso spesse volte la parola nei pubblici comizi, ma non è capace di tenere conferenze. [...] Ha sempre preso parte a manifestazioni sovversive, e fu più volte arrestata pel suo carattere ribelle"13. Lucia Minon "È persona di carattere violento, fornita di scarsa educazione e cultura, abbastanza intelligente. [...] È dedita ai lavori domestici ma nelle ore libere gradisce la lettura di romanzi e libri sovversivi". Dopo una perquisizione il verbale registra con scrupolo che "Tra le carte e documenti di nessun interesse politico le si rinvenivano due poesie: una intitolata "Inno dei socialisti anarchici" e l'altra che comincia con le parole "Addio Lugano" di carattere prettamente rivoluzionario anarchico" (ACS-CPC, b. 3304). Rita Montagnana "professa apertamente idee socialiste

12 Il pregiudizio sulla donna parte da lontano. Già nel 1487, nel Malleus maleficarum, le donne erano descritte come persone "di debole intelligenza, ciarliere, vendicative, invidiose, colleriche, volubili, smemorate, mentitrici, dai desideri insaziabili", caratteristiche che ricordano da vicino le descrizioni delle antifasciste italiane in Spagna (Verdiglione 2006: 13).

13 ACS-CPC, b. 
antimilitariste di cui ha fatto attiva propaganda durante la guerra in tutto il territorio di questa provincia ed in quella di Alessandria" e inoltre "Ha preso parte al congresso della III Internazionale Comunista di Mosca" (ACS-CPC, b. 3358). Il pregiudizio diventa ancora più marcato con Teresa Noce, di cui si afferma con scherno che: "Le sue capacità sono alquanto modeste ma suo marito l'ha sempre tenuta a galla". "In Spagna ha redatto, per qualche tempo, il giornale della brigata in lingua italiana, si è atteggiata a donna eroica e ha fatto da corriere tra il partito comunista in Francia ed ilmovimento in Spagna. Dopo l'offensiva in Catalogna, la Noce si è rifugiata a Parigi conil pretesto che era ammalata in seguito alle privazioni subite in Spagna", "Di mediocrecultura e di discreta intelligenza, di dubbia condotta morale" (ACS-CPC, b. 3553). Elettra Pollastrini "si iscrisse in una scuola di Linotipismo istituita dal comunismoper la preparazione alla propaganda ed al lavoro degli affiliati al partito", "ha lasciato l'occupazione che aveva al Bureau d'Editions [...] ma continua ad esplicare attività antifascista, specie in questi ultimi tempi, nei gruppi comunisti femminili italiani". "Si dimostra di sentimenti antifascisti, interviene a riunioni ma è da considerarsi elemento di secondaria importanza non essendo in grado di svolgere alcuna attività politica" (ACS-CPC, b. 4066). Leonetta Mazzini, arrestata in Spagna, "sembra avesse incarichi importanti presso il SRI" (ACS-CPC, b. 3181). Camilla Restellini "esplica sempre attiva propaganda antifascista, scrivendo anche articoli su giornali socialisti" (ACS-CPC, b. 4288).

Aveva ragione Le Goff, non sono documenti innocui. Le antifasciste sono letteralmente stigmatizzate, primo, per come vivono la loro sfera privata -sentimenti, cultura, istruzione, ideologia- e secondo, proprio perché si permettono di viverne una pubblica, con partecipazione alla politica e alla guerra. Si potrebbe tentare di giustificare tutto questo guardando chi stava dietro alle scrivanie, chi redigeva questi verbali, questi interrogatori, anonimi burocrati, poco abituati a pensare dal tanto ubbidire, con la mente intorpidita da troppo ordine rigore e disciplina.

Credo però che sarebbe assolutamente ingiusto sollevare chiunque dalle proprie responsabilità; non fu un uomo ma un Regime a creare un sistema di umiliazione della donna, che l'obbligava a rinunciare a ogni tipo di diritto e di libertà. La lotta che portano avanti queste donne è evidentemente duplice: una politica, contro il Fascismo, come i loro compagni uomini, e una di genere, che conducevano da sole, contro gli uomini, fascisti e purtroppo, a volte, anche antifascisti. Sbaglieremmo a immaginare un mondo antifascista in cui la donna è libera di vivere la propria femminilità al di fuori di canoni prestabiliti e imposti, ieri come oggi, quando i pregiudizi legati alle donne che si occupano di politica, di cultura o che lavorano, sono quanto mai attuali e risultano essere un'eredità contro cui bisogna, ancora, combattere. 


\section{RIFERIMENTI BIBLIOGRAFICI}

Archivio Centrale dello Stato, Casellario Politico Centrale (ACS-CPC).

Berti, G.N., "Note introduttive”, AAVV., Voci di compagni, schede di questura, Milano, Quaderni del Centro studi libertari Archivio Pinelli, 2002.

De Grazia, V., Le donne nel regime fascista, Marsilio, Venezia, 1993.

De Luna, G., Donne in oggetto. L'antifascismo nella società italiana, 1922-1939, Torino, Bollati Borighieri, 1995. Cantaluppi, A., Puppini, M., Non avendo mai preso un fucile tra le mani, Milano, Aicvas, 2014

Gissi, A., “Un percorso a ritroso: le donne al confino politico (1926-1943)”, Italia contemporanea, 226, (marzo 2002), pp. 31-60.

Guerrini, M., Donne contro, Milano, Zero in Condotta, 2013 http://www.ciusa. it/attachments/article/455/Documentazione\%20del\%20Coordinamento $\% 20$ Donne $\% 20$

dell'A.N.P.I.\%20di\%20Nuoro.pdf

http://www.vatican.va/holy_father/pius_xi/encyclicals/documents/hf_p-xi_ enc_19310515_quadragesimo- anno_it.html

Lazzari, G., Le parole del Fascismo, Roma, Argileto Editori, 1975 Loffredo, F., Politica della Famiglia, Milano, Valentino Bompiani, 1938.

Mazza, G., Maternità e infanzia in Regime fascista, Milano, Edizioni Licam, 1934. Meldini, P., Sposa e madre esemplare, Rimini-Firenze, Guaraldi Editore, 1975.

Ruinas, S., Scrittrici e scribacchine d'oggi, Roma, Accademia, 1930.

Verdiglione, A., Il Martello delle streghe. La sessualità femminile nel transfert degli inquisitori, Milano, Spirali, 2006. 